\title{
Susceptibility of severe COVID-19 patients to rhino-orbital mucormycosis fungal infection in different clinical manifestations
}

\author{
Nurettin Bayram ${ }^{1}$ (D) Cemal Ozsaygılı ${ }^{1} \cdot$ Hafize Sav $^{2} \cdot$ Yucel Tekin $^{3} \cdot$ Medine Gundogan $^{1} \cdot$ Emine Pangal $^{1}$. \\ Ayse Cicek ${ }^{1} \cdot$ Ibrahim Özcan ${ }^{4}$
}

Received: 10 February 2021 / Accepted: 6 May 2021 / Published online: 31 May 2021

(c) Japanese Ophthalmological Society 2021

\begin{abstract}
Purpose To present the different clinical manifestations of rhino-orbital mucormycosis (ROM) co-infection in severe COVID19 patients.

Study design Prospective observational clinical study

Methods Among 32,814 patients hospitalized with the diagnosis of COVID-19 between March 2020 and December 2020 in our center, eleven microbiologically confirmed ROM co-infection cases in severe COVID-19 patients were evaluated.

Results There were nine men and two women with a mean age of $73.1 \pm 7.7$ years. Eight patients had uncontrolled type 2 diabetes with a mean diagnosis duration of 12.1 \pm 4.4 years. All patients had COVID-19-associated acute respiratory distress syndrome and received corticosteroids. The mean time interval between COVID-19 diagnosis and ROM diagnosis was $14.4 \pm 4.3$ days. Seven patients $(63.6 \%)$ had orbital apex syndrome, and four patients $(36.4 \%)$ presented with orbital cellulitis. Endophthalmitis was detected in $54.5 \%$ of patients, and two of these patients developed retinoschisis. CT scan/ MRI revealed sino-orbital involvement in all patients, and three of these had cerebral involvement at initial presentation. All patients received intravenous and retrobulbar liposomal amphotericin B and had undergone radical debridement of involved sinuses. Intravitreal liposomal amphotericin B injected in patients with endophthalmitis. Despite all measures, $63.6 \%$ of patients expired.

Conclusions Severe COVID-19 is associated with a significant incidence of ROM with higher mortality rates due to immune dysregulation and the widespread use of steroids. Physicians should be aware of the possibility of this infection in patients with COVID-19. An aggressive multidisciplinary approach can help to reduce mortality.
\end{abstract}

Keywords Coronavirus disease $2019 \cdot$ Mucormycosis $\cdot$ Orbital cellulitis $\cdot$ Orbital apex syndrome $\cdot$ Endophthalmitis

Corresponding Author: Nurettin Bayram

Nurettin Bayram

drnbayram@gmail.com

1 Department of Ophthalmology, Kayseri City Training and Research Hospital, University of Health Science, 38080 Kayseri, Turkey

2 Department of Mycology, Kayseri City Training and Research Hospital, University of Health Science, Kayseri, Turkey

3 Department of Pathology, Kayseri City Training and Research Hospital, University of Health Science, Kayseri, Turkey

4 Department of Otorhinolaryngology-Head and Neck Surgery, Kayseri City Training and Research Hospital, University of Health Science, Kayseri, Turkey

\section{Introduction}

Coronavirus disease 2019 (COVID-19) is a global public health crisis associated with substantial mortality and morbidity worldwide. Although COVID-19 primarily affects the lungs, different disease complications, such as myocardial injury, arrhythmia, thromboembolic events, and immune dysregulation, are reported [1-3]. The disease affects T-helper cell responses, including cytokine release syndrome, which can open the door to co-infections [2, 3]. The risk of co-infections in patients with acute respiratory distress syndrome (ARDS), receiving broad-spectrum antibiotics, corticosteroids, and supported by invasive or non-invasive ventilation is even higher. The prevalence of co-infection was $63.64 \%$ among COVID-19 fatalities in a current study [4]. 
Mucormycosis belongs to the order Mucorales of the class Zygomycetes, which are ubiquitous in nature, especially in soil and decaying vegetation. These fungi develop rapidly and release large numbers of spores into the air. Human beings are often exposed to these fungi through inhalation. Mucormycosis is a rare acute invasive and life-threatening fungal infection that affects immunocompromised patients, including those with hematological malignancy, diabetes mellitus, systemic corticosteroid use, neutropenia, and organ transplantation [5]. Mucormycosis rarely causes infection due to the effectiveness of the human immune system. In susceptible patients, it is characterized by direct invasion with marked tissue necrosis of adjacent structures followed by rapid progression and angioinvasion from the nasal and sinus mucosa into the orbit and brain. Rhino-orbital involvement is a time-sensitive condition that must be recognized and treated promptly to avoid mortality and morbidity. Early diagnosis, control of the underlying condition with aggressive surgical debridement, and systemic and local antifungal therapies can improve prognosis.

Among various causes of morbidity and mortality in COVID-19 patients, the effect of mucormycosis has not been studied sufficiently. Herein we present a series of rhinoorbital mucormycosis (ROM) with different clinical manifestations in patients with severe COVID-19.

\section{Materials and methods}

This was a tertiary center prospective observational study. Among 32,814 patients hospitalized with the diagnosis of COVID-19 between March and December 2020, eleven microbiologically confirmed ROM co-infection cases in severe COVID-19 patients were evaluated. Severe COVID19 was defined as oxygen saturation $<93 \%$ in room air $(\mathrm{PaO} 2 / \mathrm{FiO} 2<300 \mathrm{mmHg})$, respiratory rate $>30$ breaths/ minute, and $>50 \%$ involvement of the lung parenchyma on chest computed tomography (CT) [6]. The University of Health Science, Kayseri City Training and Research Hospital Local Ethics Committee approved the study protocol (\#133325382), and it was conducted by the principles of the Declaration of Helsinki. Informed consent was obtained from all patients before including them in the study.

Patients' demographic data, clinical manifestations, underlying systemic conditions, laboratory and radiological investigations, medical treatment, and surgical interventions were analyzed. A detailed history, comprehensive ophthalmologic evaluation, otorhinolaryngological, and neurological examinations were assessed to determine the disease's extent and severity. Orbital apex syndrome was defined as the simultaneous dysfunction of the optic nerve and the cranial nerves, presenting with vision loss, ptosis, fixed and dilated pupil, and a complete internal and external ophthalmoplegia as a result of extensive orbital mucormycosis invasion to the optic canal region and the superior orbital fissure. Orbital cellulitis was defined as pain, proptosis, limitation of extraocular movements, and decreased visual acuity resulting from orbital involvement of mucormycosis.

Routine blood investigations were made, including complete blood counts, blood sugar, and serum creatinine. Diagnosis of mucormycosis was made based on a demonstration of broad aseptate hyphae with right-angled branching on $20 \%$ potassium hydroxide $(\mathrm{KOH})$ preparation of specimens obtained from the nasal cavity and/or paranasal sinuses and Sabouraud's Dextrose Agar culture reports (Fig. 1). Lactophenol cotton blue was used to stain fungal elements. Grocott-Gomori methenamine silver, periodic acid-Schiff, and hematoxylin \& eosin stains were used to show pathological changes in infected tissues (Figs. 1, 2, 3, and 4). Minimum inhibitory concentrations were determined for voriconazole and amphotericin B by gradient concentration strip and EUCAST techniques [7].

$\mathrm{CT}$ and/or magnetic resonance imaging (MRI) of paranasal sinuses, orbit, and brain were obtained to identify the disease's extent. Fundus photography and optical coherence tomography (OCT) were applied in eyes with a retina view. Ocular ultrasound was used when the patients' fundus could not be observed.

Diabetes was controlled with insulin therapy, and corticosteroids were ceased. All patients received systemic and retrobulbar (intraconal) liposomal amphotericin B. The systemic dose was $1.0 \mathrm{mg} / \mathrm{kg} /$ day, increasing a total dose of $2.5-3 \mathrm{~g}$, and the retrobulbar dose was $1 \mathrm{ml}$ of $3.5 \mathrm{mg} / \mathrm{ml}$. Intravitreal liposomal amphotericin B $(5 \mu \mathrm{g} / 0.1 \mathrm{ml})$ injections were used in patients with intraocular involvement. Repeat injections containing the same dose were performed if necessary, based on the clinical response. The maximum duration of therapy was 6 weeks. Renal functions were monitored throughout the treatment. All patients underwent functional endoscopic sinus surgery with extensive debridement of involved sinuses and sinus lavage by amphotericin $\mathrm{B}$, and specimens obtained were sent for histopathology and mycology. Repeat surgery was performed when necessary, based on the clinical course of the patients.

All ophthalmic procedures, including ophthalmologic examinations, fundus photography, OCT scans, ocular ultrasounds, and retrobulbar and intravitreal injections, were made in the ophthalmology outpatient clinic after work hours, after everyone had left the eye clinic, or in the weekends. Patients treated in intensive care units (ICUs) and required continuous oxygen came to the clinic in the company of an anesthesia technician and oxygen masks with reservoir bags connected to portable oxygen tanks (Supplementary video 1). All personnel involved in the patients' care used full protective equipment, including an N95 mask, gown, gloves, safety goggles, and face shield. 

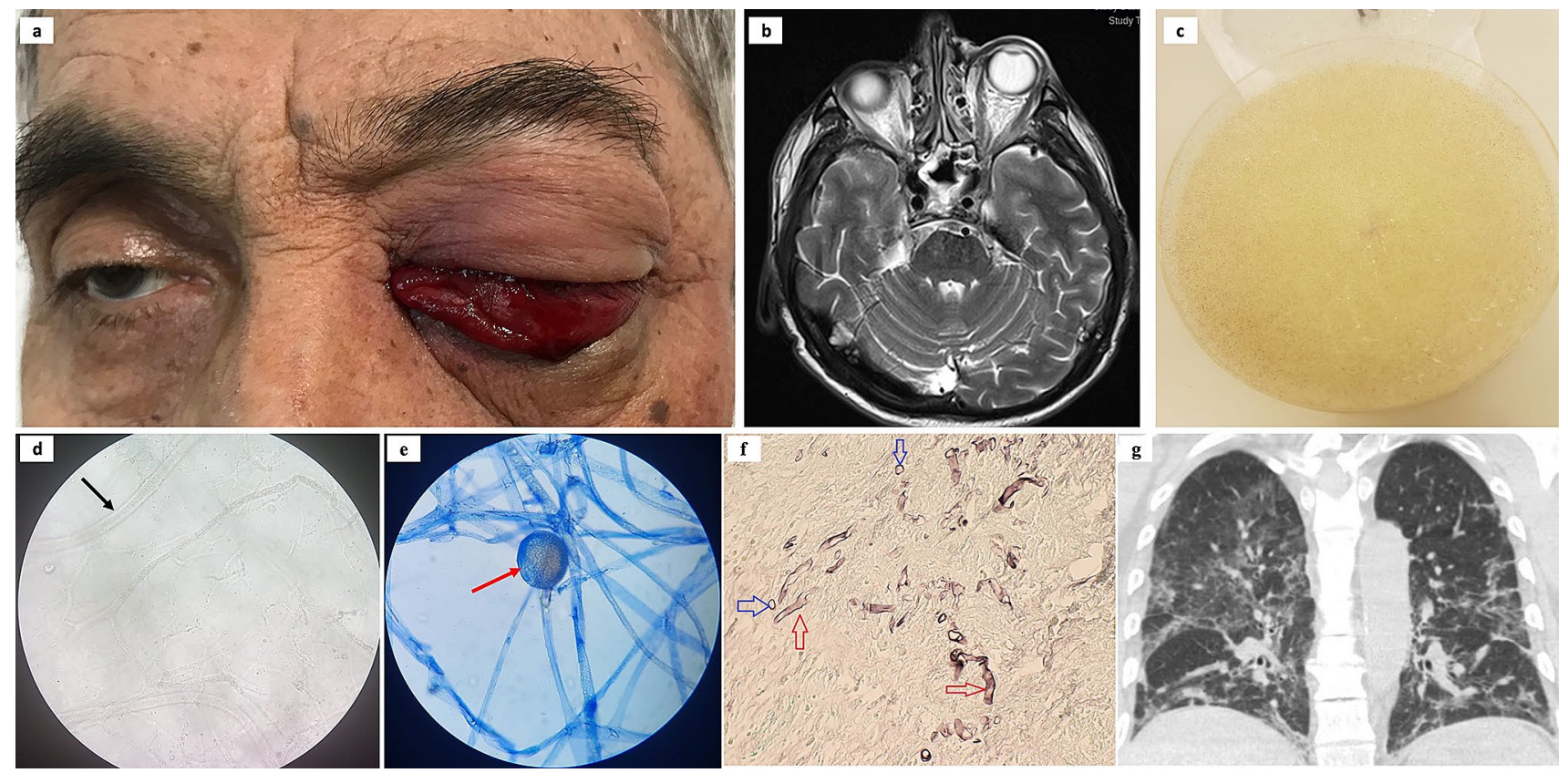

Fig. 1 a Photograph of patient \#5 with left eye orbital apex syndrome and chemosis. b Axial magnetic resonance imaging T2-weighted scan showing signs of ethmoid and sphenoid sinusitis with the involvement of left orbit. c Colony morphology of the Mucorales on Sabouraud's dextrose agar. d Potassium hydroxide mount showing broad aseptate fungal hyphae with right-angled branching (arrow). e Lactophenol cotton blue mount of the Mucorales and sporangia (arrow). f

After the procedures, the hospital's professional cleaning staff cleaned the environment and all devices based on the American Academy of Ophthalmology's disinfection recommendations [8].

The outcome for treatment was assessed in terms of treatment success and failure. Treatment success was defined as a stable and disease-free patient. Treatment failure was defined as death due to the intracranial spread of the infection.

Descriptive data were analyzed using SPSS for Mac OS version 26.0 software (SPSS, Inc.,). Continuous variables were presented as mean \pm standard deviation and range.

\section{Results}

The study included nine men and two women, with a mean age of $73.1 \pm 7.7$ years (range: $61-88$ years). Eight patients (72.7\%) had type 2 diabetes with a mean diagnosis duration of $12.1 \pm 4.4$ years (range: $7-19$ years). Three patients had chronic renal failure; another two patients developed acute renal failure; one patient received immunosuppressive therapy due to myelodysplastic syndrome. All patients had severe COVID-19-associated ARDS (Figs. 1, 2, 3, and 4) and received corticosteroids to suppress inflammation (Table 1). The patients' coagulation status showed an
Staining with Grocott-Gomori methenamine silver of invaded tissues showing the spores (blue arrows) and hyphae (red arrows). g Coronal chest computed tomography image demonstrating the common features of COVID-19 pneumonia with bilateral ground-glass and consolidative pulmonary opacities. The authors obtained the patient's publication permission by the Patient Consent Form for this figure

increase in D-Dimer, and all patients received low molecular weight heparin $(1 \mathrm{mg} / \mathrm{kg}$ body weight, equivalent to $100 \mathrm{IU})$. The mean D-Dimer level was $1362.4 \pm 468.9 \mu \mathrm{g} / \mathrm{L}$ (normal range: $220-500 \mu \mathrm{g} / \mathrm{L}$ ). Diagnosis of mucormycosis was made in all patients during the COVID-19 treatment. The mean time interval between COVID-19 diagnosis and ROM diagnosis was $14.4 \pm 4.3$ days (range: $7-23$ days). Five patients (45.5\%) were treated in the ICU due to severe COVID-19-related ARDS.

The left eye was involved in six patients $(54.5 \%)$, while the right was involved in five patients $(45.5 \%)$. The common presenting symptoms and signs were proptosis $(100 \%)$, ophthalmoplegia (63.6\%), orbital pain (81.8\%), conjunctival hyperemia or chemosis $(81.8 \%)$, ptosis $(63.6 \%)$, fixed and dilated pupil (63.6\%), vision loss (63.6\%), endophthalmitis (54.5\%), and decreased vision (27.3\%). All patients with endophthalmitis showed posterior scleritis, two developed retinochoroiditis followed by retinoschisis, and one had corneal edema (Figs. 2 and 3). The mean lag time between the onset of symptoms and ROM diagnosis was $5.1 \pm 1.8$ days (range: $2-8$ days).

The culture was positive for all patients, and lactophenol cotton blue mount revealed broad, aseptate hyphae (Fig. 1). CT scan and/or MRI demonstrated paranasal sinusitis signs with spread infection to the orbit (Figs. 1, 2, 3, and 4). The 

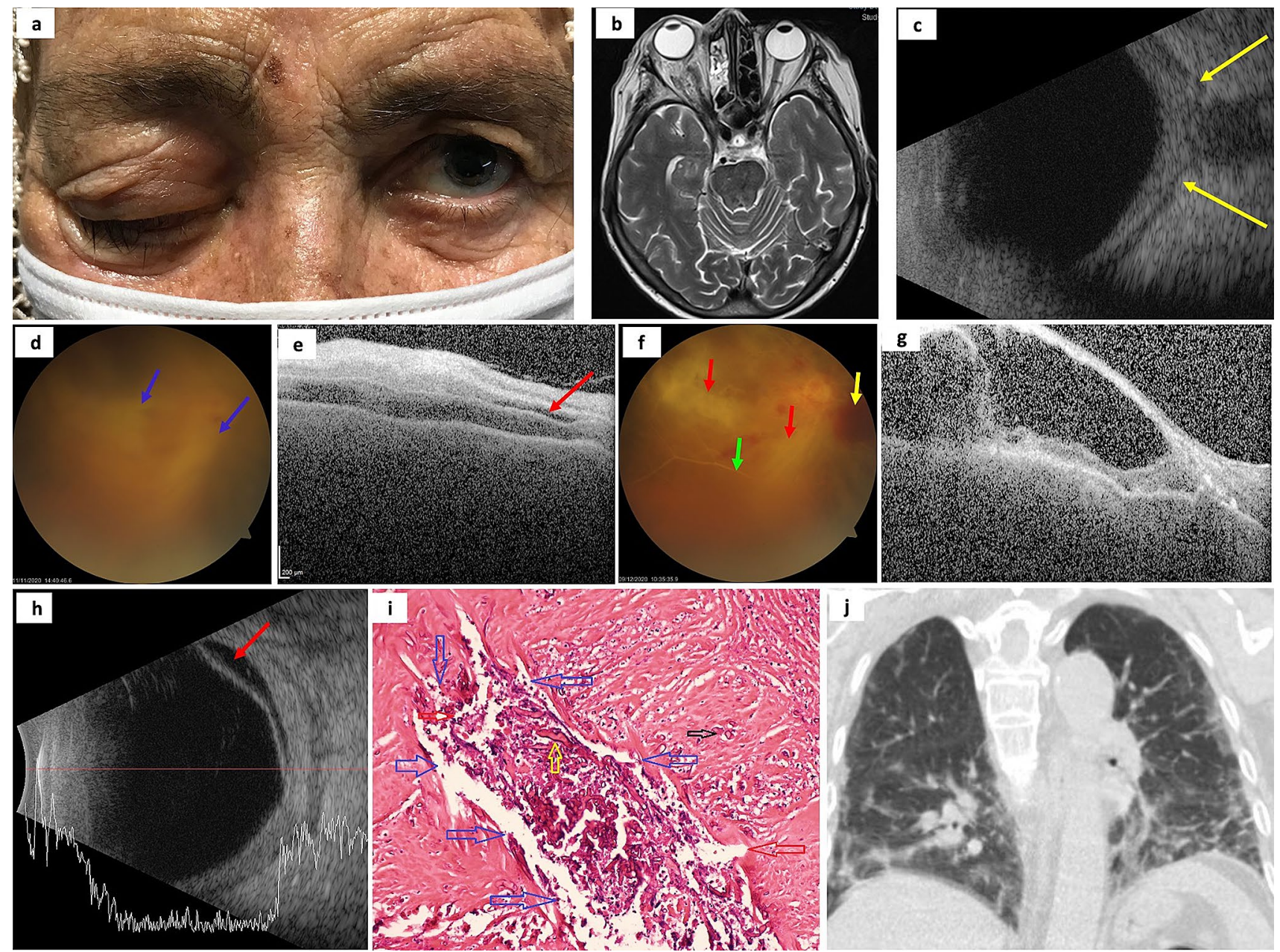

Fig. 2 a Photograph of patient \#1 with right eye orbital apex syndrome. b Axial magnetic resonance imaging T2-weighted scan showing signs of ethmoid and sphenoid sinusitis with the involvement of right orbit. c Ocular ultrasound demonstrates posterior scleritis, characterized by thickening of the posterior eyewall and increased fluid in subtenon space and around the optic nerve (" $\mathrm{T}$ " sign, yellow arrows). d Fundus photography shows two large retinochoroiditis areas (blue arrows) under severe vitritis. e Optical coherence tomography (OCT) reveals an intraretinal demarcation line between the inner nuclear layer and the outer plexiform layer with the fluid accumulated in the retinochoroiditis areas (red arrow). f After two intravitreal amphotericin $\mathrm{B}$, the visualization of the fundus is better due

ethmoid $(90.9 \%)$ and maxillary sinuses $(81.8 \%)$ were the most commonly involved, followed by sphenoid (54.5\%) and frontal (36.4\%). In total, ten patients $(90.9 \%)$ had multiple sinus involvement. Seven patients $(63.6 \%)$ developed orbital apex syndrome (Supplementary videos 1 and 2). Intracranial involvement was revealed in three patients at initial presentation, and a change in consciousness developed in one of these patients, and epileptic seizures were observed in one another.

Eight patients (72.7\%) underwent a second surgical procedure for extensive debridement of involved tissues. In to decreasing vitritis. Although retinochoroiditis areas were getting smaller (red arrows), retinal hemorrhages (yellow arrow) and empty vessels (green arrow) developed. Simultaneously, OCT (g) and ocular ultrasound (h, red arrow) demonstrate retinoschisis in the retinochoroiditis areas. i Staining with hematoxylin \& eosin of invaded tissues showing the spores (black arrow), hyphae (yellow arrow), and a cluster of both (blue arrows) with acute suppurative inflammation findings. $\mathbf{j}$ Coronal chest computed tomography image demonstrates the common features of COVID-19 pneumonia with bilateral groundglass opacities. The authors obtained the patient's publication permission by the Patient Consent Form for this figure

in-vitro susceptibility testing of Mucor spp., the minimum inhibitory concentration (MIC) of amphotericin B was lower than voriconazole (Table 1). The mean MIC of amphotericin B was $2.5 \pm 1.0 \mu \mathrm{g} / \mathrm{ml}$ (range: $1.5-4 \mu \mathrm{g} / \mathrm{ml}$ ). The MIC of voriconazole was $>32 \mu \mathrm{g} / \mathrm{ml}$ in all patients. The mean number of retrobulbar and intravitreal amphotericin B injections was $2.2 \pm 0.6$ and $2.3 \pm 0.5$, respectively. The interval between repeat injections varied from 2 to 8 days for both retrobulbar and intravitreal. The mean duration of follow-up was $51.2 \pm 41.7$ days (range: $15-153$ days). In the followup process, seven patients $(63.6 \%)$ unfortunately passed 


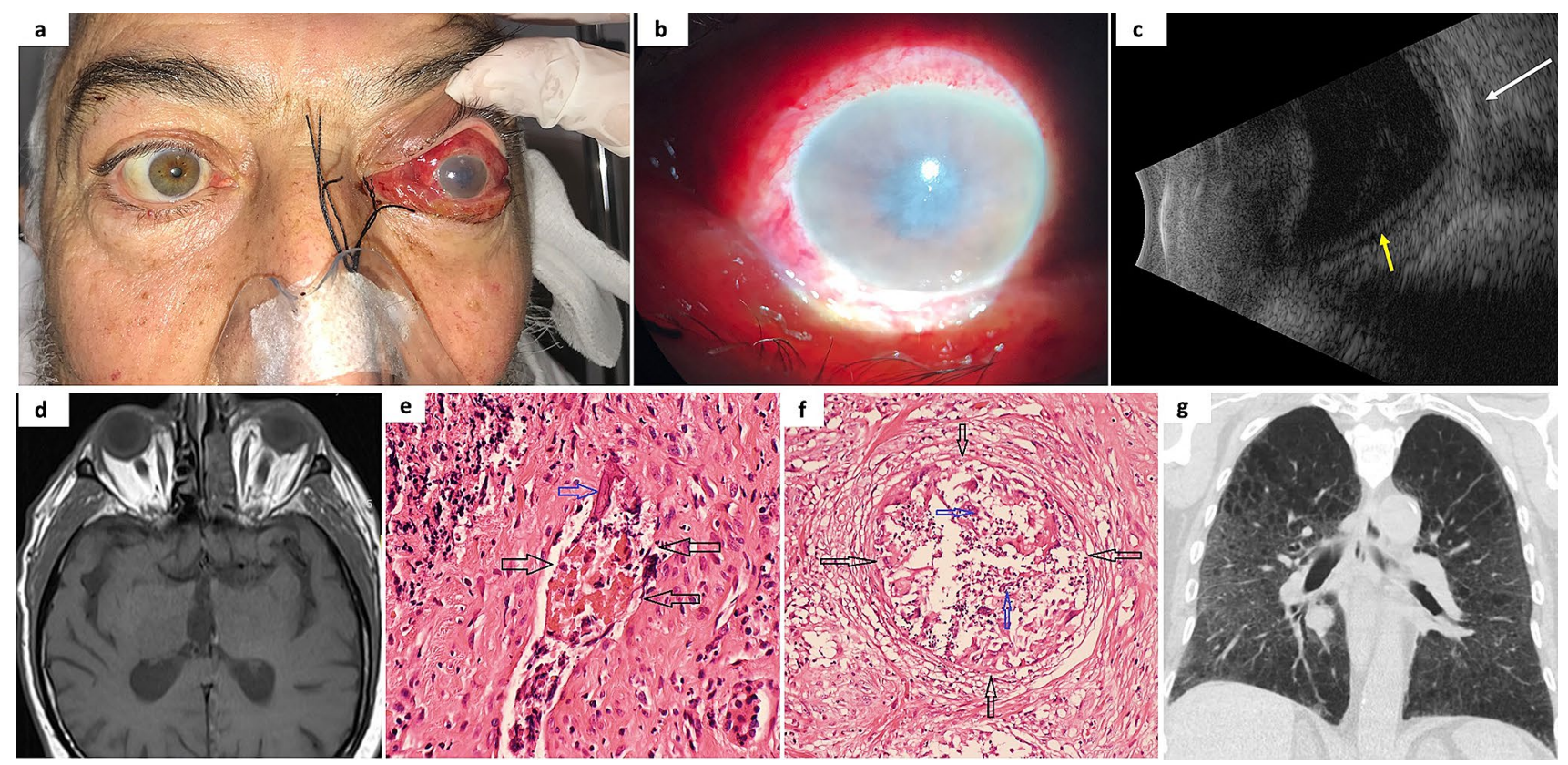

Fig. 3 a Photograph of patient \#6 with left eye orbital apex syndrome and chemosis. b Anterior segment photography shows corneal edema and a mid-dilated pupil. c Ocular ultrasound is compatible with posterior scleritis (white arrow) and retinoschisis (yellow arrow). d Axial magnetic resonance imaging T1-weighted scan showing signs of ethmoid and sphenoid sinusitis with the involvement of left orbit. e Staining with hematoxylin \& eosin of invaded tissues showing a thrombus obstructing the blood vessel lumen (black arrows) and

away. Among these patients, five expired due to intracranial dissemination of mucormycosis, and two passed away from ARDS due to COVID-19. We did not detect myocardial injury, arrhythmia, or thromboembolic events in any patient. Visual acuity improved in two patients who presented with orbital cellulitis. In these two patients' eyes, the best-corrected visual acuities were 0.40 and 1.00 LogMAR initially, and 0.30 and $0.6 \operatorname{LogMAR}$ at the last control visit, respectively.

\section{Discussion}

Although our knowledge about fungal co-infection in COVID-19 patients increases day by day [9], little is known about the impact of severe COVID-19 on ROM. Severely ill COVID-19 patients have higher pro-inflammatory cytokines, including interleukin-1 (IL-1), IL-2, IL-6, tumor necrosis factor- $\alpha$, increase in IL-4 and IL-10 levels of anti-inflammatory cytokines, less CD4 interferon- $\gamma$ expression, and fewer CD4 and CD8 cells [2, 3]. Besides the disease's immunological features, most people affected by severe COVID-19 are old and have other predisposing conditions such as type 2 diabetes mellitus. In addition to these, the patients frequently hyphae that invade the vessel wall (blue arrow), and $\mathbf{f}$ Staining with hematoxylin \& eosin of invaded tissues showing granuloma formation (black arrows), and hyphae which are broken down by inflammatory cells in the middle of the granuloma (blue arrows). g Coronal chest computed tomography image demonstrating the common features of COVID-19 pneumonia with diffuse bilateral ground-glass opacities. The authors obtained the patient's publication permission by the Patient Consent Form for this figure

receive broad-spectrum antibiotics and corticosteroids. Also, they are supported by invasive or non-invasive ventilation due to severe ARDS. As a result, critically ill COVID-19 patients are candidates for very high-risk ROM. Although there is no direct relationship between COVID-19 and mucormycosis, the findings mentioned above suggest the existence of an indirect association. In the present study population, being an average of 73 years of age, the presence of diabetes mellitus in $72.7 \%$ of the patients, the use of steroids for COVID-19-related ARDS in all patients, and the fact that $45.5 \%$ of the patients were treated in the ICU illustrate the above. While the total number of patients with ROM was six over 2 years between 2013 and 2016 in our previous study [10], having eleven patients in the last 9 months shows how much the disease incidence has increased in severe COVID19 patients. To raise awareness, we shared our study results with the physicians working in all pandemic wards and ICU in our hospital and emphasized the severity of the disease and the importance of early diagnosis.

Because Mucorales have a ketone reductase enzyme, they thrive in hyperglycemic and diabetic ketoacidosis states associated with poor prognosis. In Pub-Med research, there were only eight microbiologically confirmed ROM co-infection cases in COVID-19 patients, and all of them 


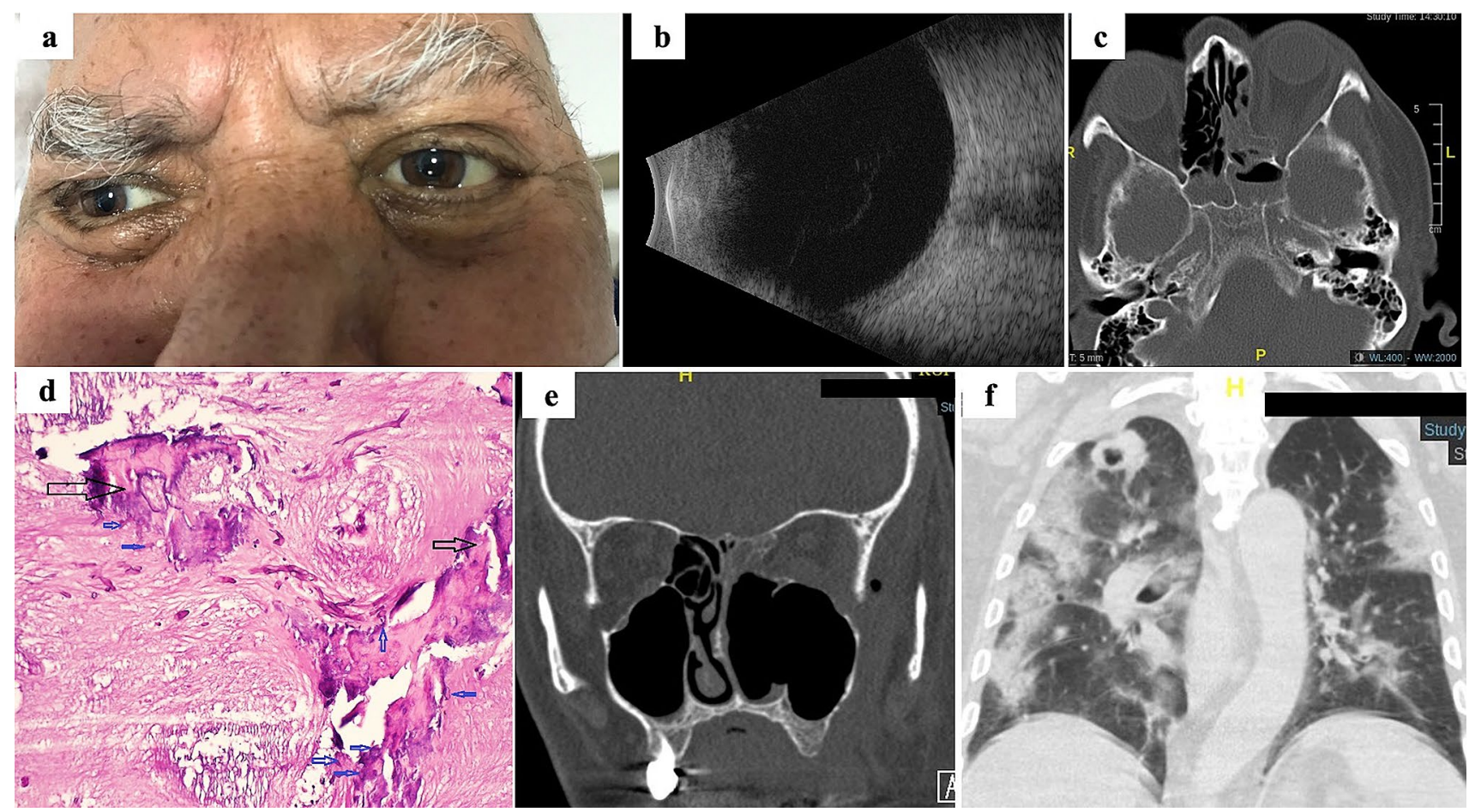

Fig. 4 a Photograph of patient \#2 with left eye orbital cellulitis. b The ocular ultrasound did not show signs of ocular involvement. c Axial computed tomography (CT) scan showing left-sided orbital cellulitis with opacification of ethmoid and sphenoid sinuses and bone destruction in the medial orbital wall. d Staining with periodic acidSchiff of invaded tissues showing hyphae infiltrations (blue arrows) in trabeculae of bone (black arrows). e In the coronal CT scan is taken

had diabetes [11-14]. These cases and our patients' clinical data show that uncontrolled diabetes or diabetic ketoacidosis can make COVID-19 patients susceptible to mucormycosis infection.

Corticosteroids not only reduce the activity of the immune system but also cause drug-induced hyperglycemia. Both of these aggravate the clinical course of the patients. Corticosteroids were immediately discontinued, and diabetes was controlled with insulin therapy to improve patients' metabolic control.

Endophthalmitis developed in six patients. Ocular ultrasound showed posterior scleritis in all endophthalmitis' patients; two developed retinochoroiditis followed by retinoschisis. OCT revealed intraretinal fluid accumulation in the retinochoroiditis area and finally resulted in retinoschisis (Fig. 2e, g). These results show that ocular involvement develops following direct invasion from the adjacent orbital structures. The most striking clinical difference in our patients was the frequency of endophthalmitis. While endophthalmitis frequency was reported as $1-6 \%$ in nonCOVID-19 studies [15, 16], it was $54.5 \%$ in our series.

CT scans and MRI demonstrated evidence of mucosal thickening, sinuses opacification, orbital and intracranial after radical sinus surgery, the left middle and inferior turbinates and the maxillary sinus medial wall are not observed. f Coronal chest CT scan demonstrating the common features of COVID-19 pneumonia with bilateral ground-glass and consolidative pulmonary opacities. The authors obtained the patient's publication permission by the Patient Consent Form for this figure

involvement. In this study, seven rhino-orbital and three patients of rhino-orbital-cerebral mucormycosis were identified by these imaging modalities. In this study, the most frequently involved sinuses were ethmoid (90.9\%), followed by maxillary sinuses $(81.8 \%)$, which is in line with the literature [17].

ROM is diagnosed by typical clinical presentation and by detecting broad aseptate hyphae with right-angled branching in $\mathrm{KOH}$ mounts (Fig. 1d). Lactophenol cotton blue is used to visualize microscopic structures better, identifying aseptate hyphae and sporangiospores (Fig. 1e). Staining with Grocott-Gomori methenamine silver best shows the hyphae in invaded tissues (Fig. 1f). Periodic acid-Schiff and hematoxylin \& eosin stains can be used to show pathological changes in tissues, including acute suppurative inflammation with focal areas of granulomatous inflammation and angioinvasion by hyphae with consequent thrombosis and infarction (Figs. 2, 3, and 4). The immunohistochemical investigation, polymerase chain reaction for fungal DNA, and in situ hybridization can be used for tissue diagnosis as alternate techniques [18].

In this study, in vitro susceptibility testing of Mucor spp. against amphotericin B and voriconazole showed that 


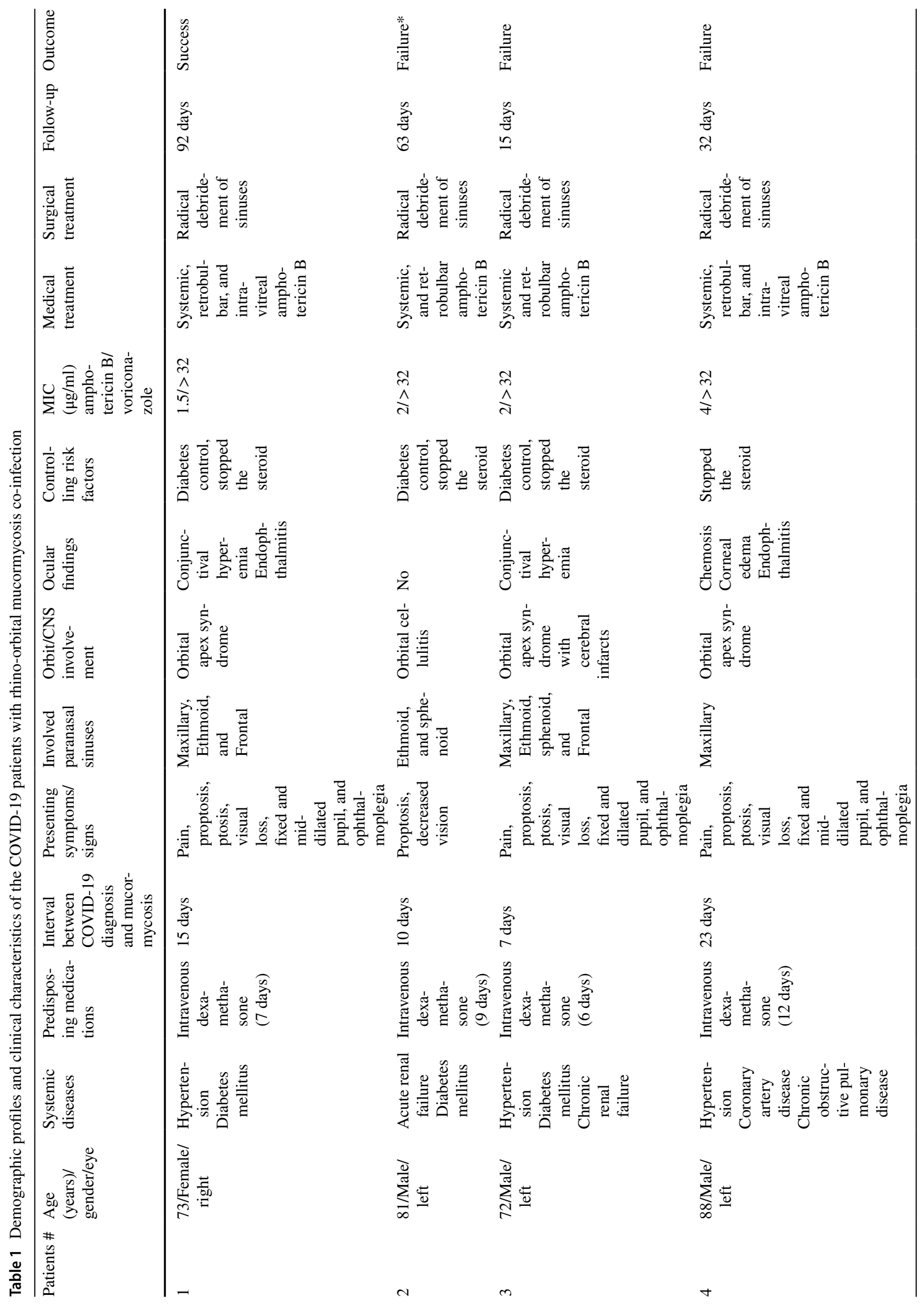




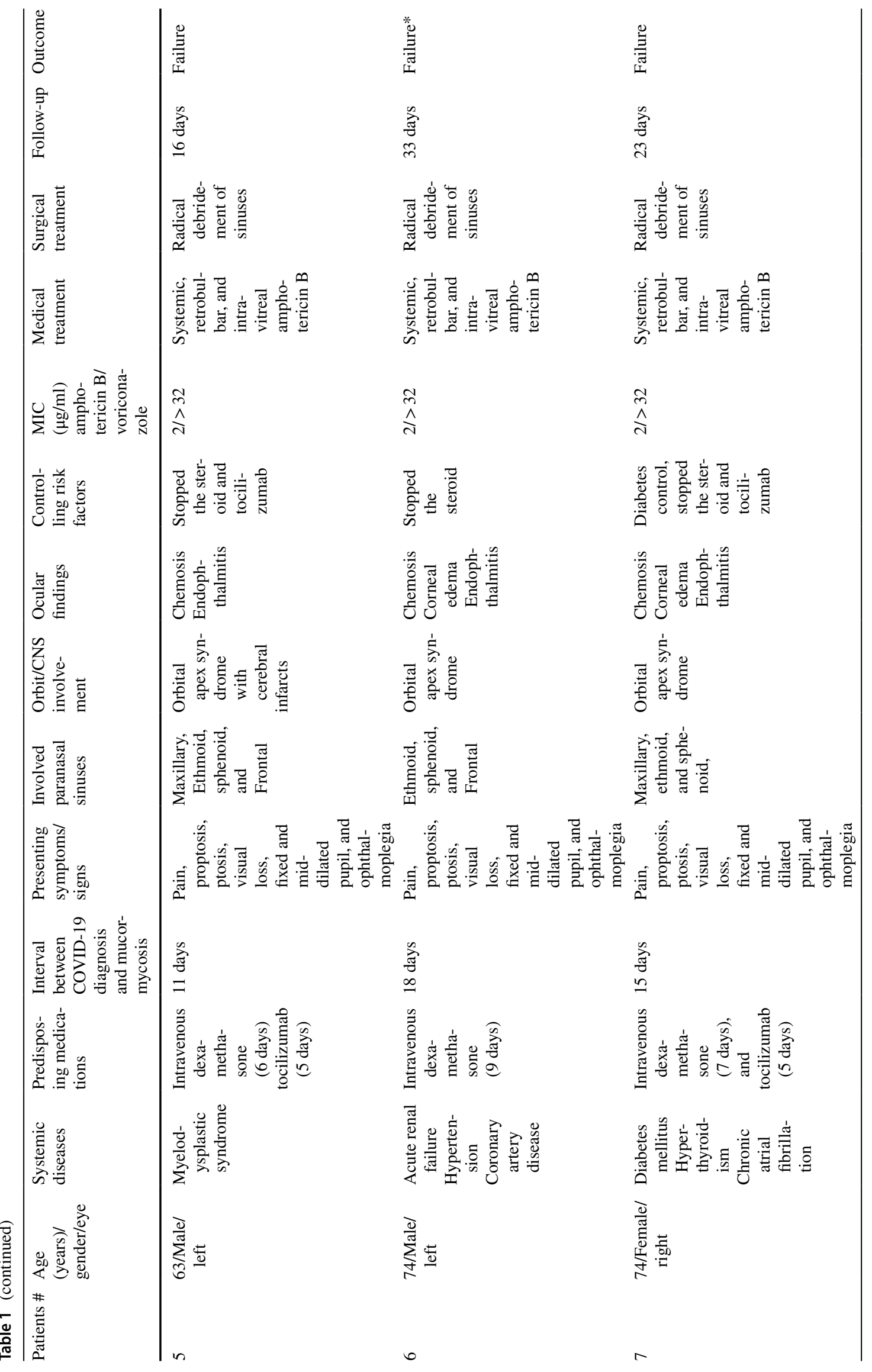




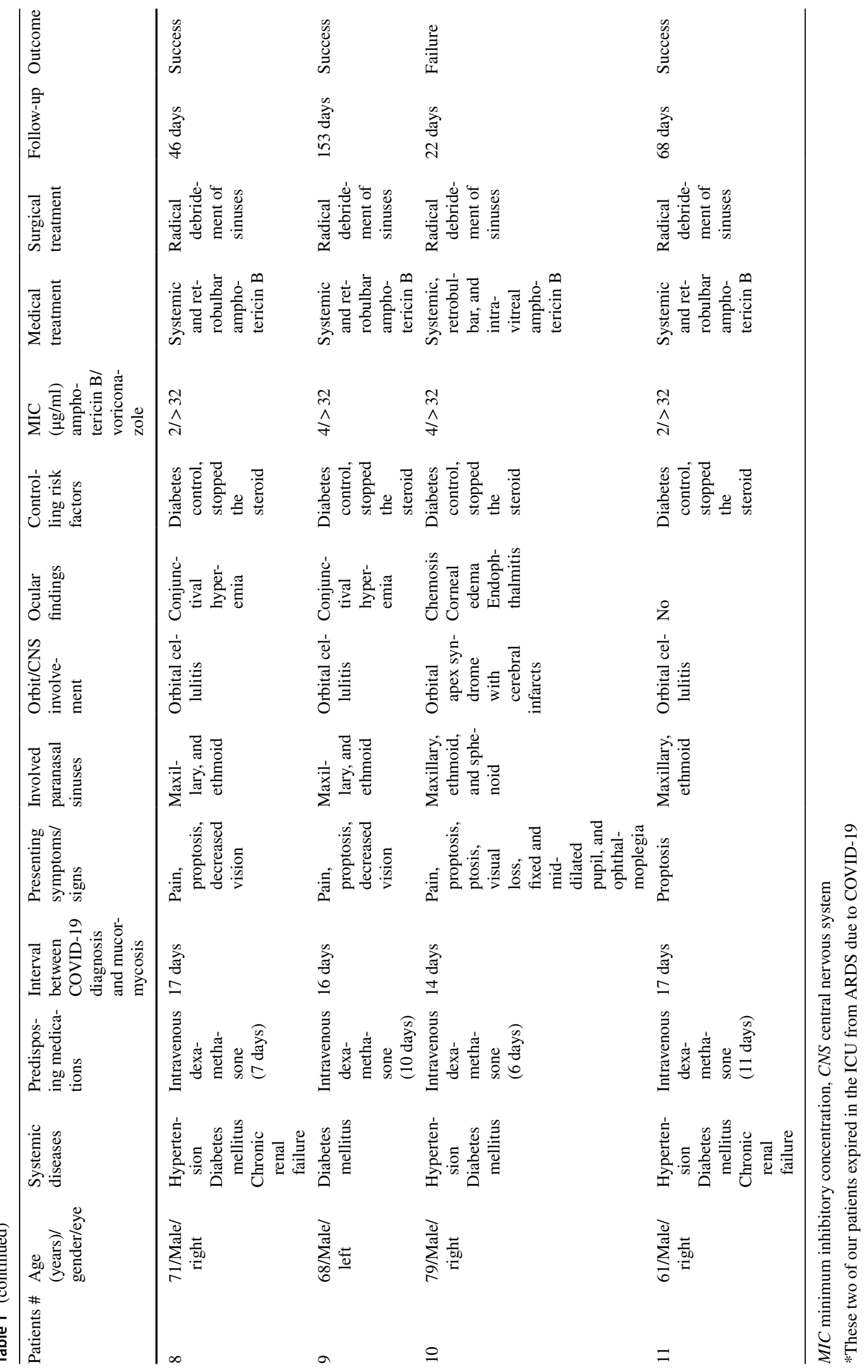


the MIC of amphotericin B was lower than voriconazole (Table 1). According to the global guideline for diagnosing and managing mucormycosis [19] and the present study results, amphotericin $B$ should be the first-line drug in mucormycosis treatment. It should be initiated as soon as the diagnosis is suspected. All our patients received systemic liposomal amphotericin B, which is more effective and less toxic in facilitating prolonged administration without side effects [20]. Mucormycosis endophthalmitis can be successfully treated with intravitreal amphotericin B [21]. Retrobulbar injection of amphotericin B should be considered an adjunctive treatment modality to prevent exenteration [22]. In our clinical practice, we use a retrobulbar injection of amphotericin B in all ROM patients and apply intravitreal amphotericin B in the presence of endophthalmitis.

Antifungal treatment alone is ineffective due to vascular thrombosis and an extensive ischemic necrosis barrier, preventing antifungal agents' entry in adequate concentrations. Therefore, radical debridement of infected and necrotic tissue with drainage of infected paranasal sinuses should be performed as soon as possible to minimize the fungal load in the tissue. All our patients developed these symptoms while being treated in the hospital, and all underwent surgery within an average of 5.1 days. Simultaneously, both systemic and retrobulbar amphotericin B treatments were given to all patients. Also, intravitreal amphotericin B treatment was given to six patients with endophthalmitis. All these treatments were repeated when necessary, according to the clinical course of the patients. As a result, the overall survival rate of our patients was $36.4 \%$. However, it should be kept in mind that two of our patients were lost from ARDS due to COVID-19. If we exclude these two patients, the survival rate in ROM was $44.4 \%$. In a previous review, including ROM in non-COVID-19 patients, the overall survival rate was 59.5\% [23]. Previous literature [11-13] and this study's outcomes show that the coexistence of ROM and severe COVID-19 infection is associated with higher mortality rates than previously reported non-COVID-19 patients. In contrast, death was not reported in a recent COVID-19 case series of six patients [14]. However, the authors did not give detailed information about the severity of COVID-19, and all, except one patient, presented after recovering from COVID-19.

This study's limitation is that our results may not reflect the exact survival rate of ROM in COVID-19 patients. Because two of our patients died in the ICU from ARDS due to COVID-19, we do not know whether they would have died due to ROM. However, even if we accept that these two patients would not have died from ROM, the survival rate would still be lower than in the previous study.

In conclusion, this study's outcomes show that ROM incidence has dramatically increased with higher mortality rates in severe COVID-19 patients. These results suggest that the prognosis of preexisting ROM may be worse after severe SARS-CoV-2 infection. SARS-CoV-2-related immune dysregulation, the widespread use of steroids/broad-spectrum antibiotics, and ventilatory support seem to be the main factors that make patients susceptible. Physicians should be aware of the possibility of this infection in patients with COVID-19. An aggressive multidisciplinary approach to ROM can help reduce mortality. Early diagnosis, control of the systemic predisposing factors, prompt initiation of systemic and retrobulbar antifungal therapy, intravitreal amphotericin B injection in patients having intraocular involvement, and radical debridement of involved sinuses are crucial to improving outcomes.

Supplementary Information The online version contains supplementary material available at https://doi.org/10.1007/s10384-021-00845-5.

Author contributions NB designed the study and prepared the manuscript. NB, CO, MG, EP, AC, and IO provided clinical care to the patient. YT made the histopathologic investigations of the tissues. HS realized mycological examinations of the tissues. All authors provide essential contributions to the revision of the study. All authors approved the final manuscript.

Conflicts of interest N. Bayram, None; C. Ozsaygili, None; H. Sav, None; Y. Tekin, None; M. Gundogan, None; E. Pangal, None; A. Cicek, None; İ. Özcan, None.

\section{References}

1. Giustino G, Pinney SP, Lala A, Reddy VY, Johnston-Cox HA, Mechanick JI, et al. Coronavirus and cardiovascular disease, myocardial injury, and arrhythmia: JACC focus seminar. J Am Coll Cardiol. 2020;76:2011-23.

2. Diao B, Wang C, Tan Y, Chen X, Liu Y, Ning L, et al. Reduction and functional exhaustion of $\mathrm{T}$ cells in patients with coronavirus disease 2019 (COVID-19). Front Immunol. 2020;11:827.

3. Tay MZ, Poh CM, Rénia L, MacAry PA, Ng LFP. The trinity of COVID-19: immunity, inflammation and intervention. Nat Rev Immunol. 2020;20:363-74.

4. Lv Z, Cheng S, Le J, Huang J, Feng L, Zhang B, et al. Clinical characteristics and co-infections of 354 hospitalized patients with COVID-19 in Wuhan, China: a retrospective cohort study. Microbes Infect. 2020;22:195-9.

5. Hirabayashi KE, Idowu OO, Kalin-Hajdu E, Oldenburg CE, Brodie FL, Kersten RC, et al. Invasive fungal sinusitis: risk factors for visual acuity outcomes and mortality. Ophthalmic Plast Reconstr Surg. 2019;35:535-42.

6. Wu Z, McGoogan JM. Characteristics of and important lessons from the coronavirus disease 2019 (COVID-19) outbreak in China: summary of a report of 72314 cases from the Chinese center for disease control and prevention. JAMA. 2020;323:1239-42.

7. Arendrup MC, Cuenca-Estrella M, Lass-Flörl C, Hope W, Howard SJ, Subcommittee on Antifungal Susceptibility Testing (AFST) of the ESCMID European Committee for Antimicrobial Susceptibility Testing (EUCAST). Method for the determination of broth dilution minimum inhibitory concentrations of antifungal agents for conidia forming moulds. EUCAST definitive document E. Def 9.2; Basel, Switzerland: 2014. Available online: https://eucast.org/ 
fileadmin/src/media/PDFs/EUCAST_files/AFST/Files/EUCASTAFST_EDEF_9_2_Mould_testing_20140815.pdf. Accessed 29 Jan 2021

8. American Academy of Ophthalmology. Important coronavirus updates for ophthalmologists. 2020. Available at https://www. aao.org/headline/alert-important-coronavirus-context\#resuming. Accessed 31 Mar 2021

9. Pemán J, Ruiz-Gaitán A, García-Vidal C, Salavert M, Ramírez P, Puchades F, et al. Fungal co-infection in COVID-19 patients: should we be concerned? Rev Iberoam Micol. 2020;37:41-6.

10. Sağıt M, Hira İ, Halil Polat H, Beştepe Dursun Z, Çelik İ, Sav $\mathrm{H}$, et al. Our therapeutic approach in patients with rino-orbital mukormikozis. KBB-Forum. 2017;16:35-42.

11. Mehta S, Pandey A. Rhino-orbital mucormycosis associated with COVID-19. Cureus. 2020;12:e10726.

12. Mekonnen ZK, Ashraf DC, Jankowski T, Grob SR, Vagefi MR, Kersten RC, et al. Acute invasive rhino-orbital mucormycosis in a patient with COVID-19-associated acute respiratory distress syndrome. Ophthalmic Plast Reconstr Surg. 2021;37:e40-80.

13. Werthman-Ehrenreich A. Mucormycosis with orbital compartment syndrome in a patient with COVID-19. Am J Emerg Med. 2021;42:264.e5-264.e8

14. Sen M, Lahane S, Lahane TP, Parekh R, Honavar SG. Mucor in a viral land: a tale of two pathogens. Indian J Ophthalmol. 2021;69:244-52.

15. Bhansali A, Bhadada S, Sharma A, Suresh V, Gupta A, Singh P, et al. Presentation and outcome of rhino-orbital-cerebral mucormycosis in patients with diabetes. Postgrad Med J. 2004;80:670-4.

16. Yohai RA, Bullock JD, Aziz AA, Markert RJ. Survival factors in rhino-orbital-cerebral mucormycosis. Surv Ophthalmol. 1994;39:3-22.

17. Gupta S, Goyal R, Kaore NM. Rhino-orbital-cerebral mucormycosis: battle with the deadly enemy. Indian J Otolaryngol Head Neck Surg. 2020;72:104-11.
18. Guarner J, Brandt ME. Histopathologic diagnosis of fungal infections in the 21st century. Clin Microbiol Rev. 2011;24:247-80.

19. Cornely OA, Alastruey-Izquierdo A, Arenz D, Chen SCA, Dannaoui E, Hochhegger B, et al. Global guideline for the diagnosis and management of mucormycosis: an initiative of the European Confederation of Medical Mycology in cooperation with the Mycoses Study Group Education and Research Consortium. Lancet Infect Dis. 2019; 19:e405-21.

20. Fisher EW, Toma A, Fisher PH, Cheesman AD. Rhinocerebral mucormycosis: use of liposomal amphotericin B. J Laryngol Otol. 1991;105:575-7.

21. Ho HC, Liew OH, Teh SS, Hanizasurana H, Ibrahim M, Shatriah I. Unilateral rhino-orbital-cerebral mucormycosis with contralateral endogenous fungal endophthalmitis. Clin Ophthalmol. 2015;9:553-6.

22. Safi M, Ang MJ, Patel P, Silkiss RZ. Rhino-orbital-cerebral mucormycosis (ROCM) and associated cerebritis treated with adjuvant retrobulbar amphotericin B. Am J Ophthalmol Case Rep. 2020;19:100771.

23. Vaughan C, Bartolo A, Vallabh N, Leong SC. A meta-analysis of survival factors in rhino-orbital-cerebral mucormycosishas anything changed in the past 20 years? Clin Otolaryngol. 2018;43:1454-64.

Publisher's Note Springer Nature remains neutral with regard to jurisdictional claims in published maps and institutional affiliations. 